Research Paper

\title{
Penetration of Endothelial Cell Coated Multicellular Tumor Spheroids by Iron Oxide Nanoparticles
}

\author{
Don N. Ho ${ }^{1,2}$, Nathan Kohler ${ }^{1}$, Aruna Sigdel ${ }^{1}$, Raghu Kalluri ${ }^{3}$, Jeffrey R. Morgan ${ }^{\circledR}{ }^{\bowtie}$, Chenjie $X u^{1,4 \bowtie}$, \\ Shouheng Sun ${ }^{1 凶}$ \\ 1. Department of Chemistry, Brown University, Providence, RI 02912 \\ 2. Center of Biomedical Engineering, Brown University, Providence, RI 02912; \\ 3. Department of Biological Chemistry and Molecular Pharmacology, Harvard-MIT Division of Health Sciences and Technology, Depart- \\ ment of Medicine Beth Israel Deaconess Medical Center, Harvard Medical School, Boston, MA 02114, USA \\ 4. Department of Medicine, Brigham and Women's Hospital, Harvard Medical School, Harvard Stem Cell Institute, Harvard-MIT, Division \\ of Health Sciences and Technology, 65 Landsdowne Street, Cambridge, MA 02139, USA
}

$\checkmark$ Corresponding author: Email: Jeffrey_Morgan@Brown.edu; chjxu@mit.edu; ssun@brown.edu

(c) Ivyspring International Publisher. This is an open-access article distributed under the terms of the Creative Commons License (http://creativecommons.org/ licenses/by-nc-nd/3.0/). Reproduction is permitted for personal, noncommercial use, provided that the article is in whole, unmodified, and properly cited.

Received: 2011.09.28; Accepted: 2011.10.24; Published: 2012.01.01

\begin{abstract}
Iron oxide nanoparticles are a useful diagnostic contrast agent and have great potential for therapeutic applications. Multiple emerging diagnostic and therapeutic applications and the numerous versatile parameters of the nanoparticle platform require a robust biological model for characterization and assessment. Here we investigate the use of iron oxide nanoparticles that target tumor vasculature, via the tumstatin peptide, in a novel three-dimensional tissue culture model. The developed tissue culture model more closely mimics the in vivo environment with a leaky endothelium coating around a glioma tumor mass. Tumstatin-iron oxide nanoparticles showed penetration and selective targeting to endothelial cell coating on the tumor in the three-dimensional model, and had approximately 2 times greater uptake in vitro and 2.7 times tumor neo-vascularization inhibition. Tumstatin provides targeting and therapeutic capabilities to the iron oxide nanoparticle diagnostic contrast agent platform. And the novel endothelial cell-coated tumor model provides an in vitro microtissue environment to evaluate nanoparticles without moving into costly and time-consuming animal models.
\end{abstract}

Key words: iron oxide nanoparticles, tumstatin, tumor penetration, multicellular tumor spheroids, theranostic, magnetic, nanoparticle, imaging, drug delivery, anti-angiogenesis

\section{Introduction}

Iron oxide nanoparticles $\left(\mathrm{Fe}_{3} \mathrm{O}_{4} \mathrm{NPs}\right)$ hold great promise for realizing early tumor diagnosis and therapy, as well as simultaneous monitoring of the therapeutic response $[1,2]$. The efficacy of imaging and therapy of these $\mathrm{Fe}_{3} \mathrm{O}_{4} \mathrm{NPs}$ is typically evaluated by direct observation of their bio-distribution (especially with tumor selectivity), their effects on the quality of life (body weight, tumor size, etc.) and longevity in animal models. Among many evaluation parameters, efficient transport of NPs into tumor tissue in vivo is a critical aspect. Ideally, systemically-administered NPs can preferentially accumulate at tumor sites by the enhanced permeability and retention (EPR) effect, thus reducing toxicity to normal tissue [3]. However, systemically-administered NPs are often subject to a heterogeneous distribution in the tumor tissue and tend to concentrate near the tumor vasculature $[4,5]$. This is a result from the increased 
interstitial fluid pressure and the dense extracellular matrix (ECM) found in the in vivo tumor environment. As intervascular distances in tumors are relatively large $(>100 \mu \mathrm{m})$ [6], this undesired distribution limits the NP delivery and reduces their therapeutic efficacy [5].

Recently, there are emerging efforts to improve NP penetration via a multistage delivery system [7], or by grafting NPs with tumor penetration peptides [8]. Unfortunately, the verification of these platforms relies heavily on the in vivo models, which are costly, complicated, and time consuming. Given the numerous possible NP designs, it is also unrealistic to test all types of NPs with in vivo models. Thus, an in vitro system that mimics solid tumor properties is highly desired. Multicellular tumor spheroid (MTS), a three-dimensional cluster of cancer cells grown in vitro, mimics the early avascular stage of tumor growth such as the presence of ECM and diffusion gradients of nutrients and wastes $[9,10]$. As a result, MTS can contain heterogeneous regions of tumor cell growth including a necrotic center with proliferating cells restricted to the outer rim of the MTS [11]. Their similarities with tumors in vivo have provided an important platform for nanotechnologists to screen various parameters of NPs and to identify those parameters with optimal tumor penetration [12-15]. For example, Goodman et al. examined the penetration of polystyrene NPs $(20,40,100$ or $200 \mathrm{~nm})$ in SiHa MTS ( 500 um diameter) with and without collagenase treatment $[13,14]$. These pioneering studies revealed the potential of MTS as a cost-effective and time-efficient tool for examining NP penetration in deep regions of tumors.

However, MTS models in those studies were limited by their non-uniformity and simplicity. Specifically, MTS size in those studies are heterogeneous, ranging from a few millimeters to hundreds of micrometers, which presents challenges for standardization and comparison of works from different researchers. Furthermore in a biological environment, NPs must extravasate through a leaky vascular endothelium before penetrating into the solid tumor. This added parameter further decreases the transmigration efficiency of NPs and limits the subsequent penetration depth. Unfortunately, this important factor is not present in many MTS studies. Therefore, a uniform in vitro tumor model comprising of both tumor spheroids and a vascular endothelium is highly desired to study the extravasation and penetration of NPs for imaging and therapeutic purposes.

Here, we report an in vitro uniform MTS system with vascular endothelium for the extravasation and penetration studies of $\mathrm{Fe}_{3} \mathrm{O}_{4}$ NPs. We used mi- cromolded non-adhesive agarose gels [16] to construct a uniform MTS coated with endothelial cells. Utilizing the micromold and self-assembly techniques, cell-cell sorting interactions formed a model for a tumor core that must be accessed through a leaky vasculature. Rat RG2 cells, a glioblastoma model cell line, formed a MTS core similar to that of a solid tumor, while bovine-pulmonary arterial endothelial (BPAE) cells assembled on the surface of the MTS. The layer of endothelial cells restricted access to the tumor core and acted as the vascular endothelium. This technique formed hundreds of uniform replicates for the endothelium-coated tumor model in a single culture well. We demonstrate the use of this three-dimensional cell-culture platform with $\mathrm{Fe}_{3} \mathrm{O}_{4}$ NPs conjugated with tumstatin peptide, a naturally occurring fragment of type IV collagen. $\mathrm{Fe}_{3} \mathrm{O}_{4} \mathrm{NPs}$ are bio-compatible contrast agents for $\mathrm{T}_{2}$-based magnetic resonance imaging (MRI) [17], while tumstatin peptide binds specifically to $\alpha_{v} \beta_{3}$ integrin on the endothelial cells and can significantly inhibit endothelial cell proliferation [18]. A component of basement membrane, tumstatin acts upon the mTOR pathway inhibiting protein synthesis in endothelial cells. We examine the active T7 fragment (74-98) of tumstatin (3020.85 dalton). The coupling of tumstatin onto $\mathrm{Fe}_{3} \mathrm{O}_{4}$ NPs allowed the specific targeting to BPAE cells and inhibited the proliferation of these cells $[19,20]$. The penetration depth of tumstatin- $\mathrm{Fe}_{3} \mathrm{O}_{4} \mathrm{NPs}$ was visualized and quantified by fluorescent confocal imaging and transmission electron microscope (TEM) imaging. Our results establish a uniform in vitro tumor model comprising both tumor spheroid and endothelium that can be used for NP penetration studies. With the help of this model, we demonstrated the specific targeting of tumstatin modified $\mathrm{Fe}_{3} \mathrm{O}_{4}$ NPs to endothelium. We believe this work offers new opportunities for the study of NPs penetration.

\section{Materials and methods}

All chemicals and solvents except mentioned specifically were of analytical grade from Sigma-Aldrich (St Louis, MO) and were used without further purification. MTS micro-molds were obtained from MicroTissues, Inc, (Providence, RI). Tumstatin was generously donated by Dr. Raghu Kalluri. Rat RG2 glioblastoma cells and BPAE cells (CCL-209) were obtained from the American Type Culture Collection (Rockville, MD). Both cells are maintained in Dulbecco's modified Eagle's medium (DMEM, Invitrogen), supplemented with 10\% FBS (Invitrogen), 2mM L-glutamine (Invitrogen), and 1\% Pen/Strep (Invitrogen). 


\section{Synthesis and modification of $\mathrm{Fe}_{3} \mathrm{O}_{4} \mathrm{NPs}$}

$10 \mathrm{~nm} \mathrm{Fe}_{3} \mathrm{O}_{4} \mathrm{NPs}$ were synthesized through the high temperature decomposition of iron (III) acetylacetonate $\left(\mathrm{Fe}(\mathrm{acac})_{3}\right)$ in a mixture of benzyl ether and oleylamine. The synthesized NPs were made hydrophilic and functionalized through ligand exchange with dopamine modified polyethylene glycol (DPA-PEG-COOH). Detailed methods are described in our previous publications [21].

Tumstatin labeling with rhodamine B isothiocyanate (Rhod) and subsequent coupling with $\mathrm{Fe}_{3} \mathrm{O}_{4} \mathrm{NPs}$

Tumstatin was incubated with Rhod (Invitrogen) in $\mathrm{Na}_{2} \mathrm{CO}_{3} / \mathrm{NaHCO}_{3}(\mathrm{pH}=9)$ buffer at room temperature for 1 hour (Tumstatin:Rhod = 1:10). The final conjugate was purified by removing the free Rhod through the PD-10 desalting column (GE Healthcare Corp.). Then, $\mathrm{Fe}_{3} \mathrm{O}_{4} \mathrm{NPs}$ coated with DPA-PEG-COOH $(100 \mu \mathrm{g})$ were activated with ethyl(dimethylaminopropyl) carbodiimide (EDC) (100ng) and n-hydroxysuccinimide (NHS) (100 ng) in $500 \mu \mathrm{L}$ phosphate buffered saline (PBS). Then $100 \mu \mathrm{g}$ tumstatin-Rhod in $100 \mu \mathrm{L}$ PBS was mixed with $\mathrm{Fe}_{3} \mathrm{O}_{4}$ NPs for 2 hours. Tumstatin coated $\mathrm{Fe}_{3} \mathrm{O}_{4}$ NPs were purified from free tumstatin through high speed centrifugation (8000 rpm for $15 \mathrm{~min}$ ).

\section{Characterization of tumstatin- $\mathrm{Fe}_{3} \mathrm{O}_{4} \mathrm{NPs}$}

Tumstatin- $\mathrm{Fe}_{3} \mathrm{O}_{4}$ NPs were visualized through Philips CM20 at $120 \mathrm{kV}$. The hydrodynamic diameters of NPs were measured through Malvern Zeta Sizer S90. The conjugation of tumstatin onto $\mathrm{Fe}_{3} \mathrm{O}_{4} \mathrm{NPs}$ was confirmed through infrared spectroscopy (IR) by a Bruker Tensor 27 FTIR.

\section{BPAE viability test with tumstatin- $\mathrm{Fe}_{3} \mathrm{O}_{4} \mathrm{NPs}$}

BPAE cells were seeded in 96-well plates and incubated with NPs under various Fe concentrations for 24 hours. The viability was assessed through colorimetric cell viability assay (MTT assay).

\section{BPAE proliferation test with tumstatin- $\mathrm{Fe}_{3} \mathrm{O}_{4}$ NPs}

200,000 BPAE cells were seeded in T25 flasks and incubated with NPs (in triplicate) under Fe concentration at $0.1 \mathrm{mg} / \mathrm{ml}$. At 1, 3, 6, and 8 days, BPAE cells were trypsinized and counted.

\section{Tumstatin- $\mathrm{Fe}_{3} \mathrm{O}_{4}$ NPs uptake with BPAE and RG2 cells}

BPAE and RG2 cells were seeded in T25 flasks with 200,000 seeding density 24 hours before the experiments. Then the medium was replaced with $1 \%$ FBS-containing DMEM supplemented with tum- statin- $\mathrm{Fe}_{3} \mathrm{O}_{4} \mathrm{NPs}$ or $\mathrm{Fe}_{3} \mathrm{O}_{4} \mathrm{NPs}$. After 4 hours incubation, the cells were washed with PBS twice and digested with a mixture of nitric acid and hydrogen peroxide (3:1). Fe concentration was quantified with inductively coupled plasma (ICP) atomic emission spectroscopy (Jobin Yvon JY2000).

\section{Preparation of BPAE coated RG2 MTS}

Micromolds (MicroTissues, Inc) were prepared as previously described and were autoclave sterilized [16]. Autoclave-sterilized powder Ultrapure ${ }^{\mathrm{TM}}$ Agarose (Invitrogen, Carlsbad, California) was dissolved with heating in sterile water to $2 \% \mathrm{w} / \mathrm{v}$ and pipetted into the micromolds. After setting, micromolded agarose gels were separated from the mold, transferred to six-well tissue culture plates, and equilibrated overnight in culture medium. To form MTS, RG2 and BPAE cells were trypsinized, counted, and re-suspended to 500,000 cells per $200 \mu \mathrm{L}$ of culture medium at a ratio of 1:1 RG2:BPAE. Medium was removed from the tissue culture plates containing the micromolded hydrogels, and $200 \mu \mathrm{L}$ cell suspension was added dropwise with a micropipette to the center of the seeding chamber of each gel. Cells were allowed to settle into the recesses for $30 \mathrm{~min}$ before 5 $\mathrm{mL}$ of additional culture medium was carefully added to the medium exchange ports of the micromolded gels. Cells self-assembled within $24 \mathrm{~h}$ at $37^{\circ} \mathrm{C}$. MTS were maintained in the incubator and culture medium was changed every day. Side view microscopy was obtained as described in previous publications [22].

\section{Live-cell fluorescent staining of MTS with tum- statin- $\mathrm{Fe}_{3} \mathrm{O}_{4}$ visualized by confocal microscopy}

MTS were cultured in non-adhesive hydrogel for 24 hours and were incubated with culture medium containing Rhod-labeled tumstatin- $\mathrm{Fe}_{3} \mathrm{O}_{4} \mathrm{NPs}(0.1 \mathrm{mg}$ $\mathrm{Fe} / \mathrm{mL}$ ) for 4 hours, and then washed twice with PBS. $\mathrm{Fe}_{3} \mathrm{O}_{4}$ NPs labeled with Rhod but without tumstatin were used as a control. MTS were visualized on $1 \mathrm{mi}-$ cron cross-section planes by confocal microscope (Leica TCS SP2 AOBS spectral confocal microscope).

The sorting of glioma and endothelial cell heterotypic microtissues were visualized with staining by fluorescent dyes, CellTracker Red CMTPX and CellTracker Green CMFDA (Invitrogen, Carlsbad, California). Cells were loaded 1 day prior to seeding. Subconfluent flasks were incubated with DMEM containing $2.5 \mu \mathrm{M}$ CellTracker for $45 \mathrm{~min}$ at $37^{\circ} \mathrm{C}$. Dye containing DMEM was then discarded and replaced with fresh culture medium. Stained cells were trypsinized and seeded into hydrogels with a 1:1 ratio of endothelial cell and glioma cells. CellTracker Red CMTPX and CellTracker Green CMFDA were imaged 
by Leica TCS SP2 AOBS spectral confocal microscope (Leica, Wetzlar, Germany) or epi-fluorescent microscopy using a model (Olympus, Tokyo, Japan) with an excitation/detection of $577 / 602 \mathrm{~nm}$ and $492 / 517 \mathrm{~nm}$, respectively.

\section{TEM preparation of MTS labeled with tum- statin- $\mathrm{Fe}_{3} \mathrm{O}_{4}$}

MTS were cultured in non-adhesive hydrogels for 24 hours and were incubated with culture medium containing Rhod-labeled tumstatin- $\mathrm{Fe}_{3} \mathrm{O}_{4} \mathrm{NPs}(0.1 \mathrm{mg}$ $\mathrm{Fe} / \mathrm{mL}$ ) for 24 hours, and then washed twice with PBS. MTS were fixed in phosphate-buffered Karnofsky's solution and hydrogels were inverted to isolate MTS. Collected MTS were suspended in fixative for 30 minutes before they were spun down using centrifugation and fixative was aspirated. MTS were stained with $2 \%$ osmium tetroxide at $4^{\circ} \mathrm{C}$ overnight, followed by dehydration using graded ethanol and embedded in Epon 812 resin. Resin blocks were sectioned using a microtome, doubly stained with uranyl acetate and lead hydroxide, and imaged on TEM (Philips CM20 at 80kV).

\section{SEM preparation of MTS labeled with tum- statin- $\mathrm{Fe}_{3} \mathrm{O}_{4}$}

MTS were cultured in non-adhesive hydrogels for 24 hours and were fixed in phosphate-buffered Karnofsky's solution. Hydrogels were inverted to isolate MTS. Collected MTS were suspended in fixative for 30 minutes before they were spun down using centrifugation and fixative was aspirated. MTS were dehydrated using graded ethanol and transferred to the critical point drying apparatus for final substitution with liquid $\mathrm{CO}_{2}$. Dried MTS were placed on carbon adhesive SEM tabs and spin-coated for observation and imaging in SEM (Hitachi 2700).

\section{Spheroid immunostaining}

MTS cultured in non-adhesive hydrogels for 24 hours were fixed in phosphate-buffered Karnofsky solution ( $2 \%$ paraformaldehyde, $2 \%$ glutaraldehyde). Gels were inverted to remove MTS. MTS were washed five times in $0.002 \%$ Triton X-100 in phosphate buffered solution (PBS), and MTS were gently agitated for five minutes between washes. Cells in the MTS were then permeablized for one hour in $0.5 \%$ Triton X-100 in PBS. MTS were washed twice in PBS, then blocked in 1\% BSA for 20 minutes. $10 \mu \mathrm{L}$ Oregon Green $488^{\circledR}$-conjugated phalloidin (Invitrogen, Carlsbad, California) in $\mathrm{MeOH}$ was dissolved in $400 \mu \mathrm{L}$ PBS and incubated for 30 minutes. MTS were washed twice in PBS and blocked with $1 \%$ BSA and $4 \%$ goat serum. $2 \mathrm{~mL}$ of $20 \mu \mathrm{g} / \mathrm{mL}$ of primary antibody (mouse anti-occludin and rabbit anti-zonula occluden 1, Invi- trogen, Carlsbad, California) in working solution ( $4 \%$ goat serum, 1\% BSA, in PBS) was added and incubated 8 hours overnight at $4{ }^{\circ} \mathrm{C}$. Primary antibody was removed and blocking solution (working solution, $0.1 \%$ Triton in PBS) was added for one hour. MTS were rinsed three times in one $\mathrm{mL} 20 \mu \mathrm{g} / \mathrm{mL}$ of PBS and secondary antibody (Texas Red-X goat anti-mouse and Alexa Fluor 546 goat anti-rabbit, Invitrogen, Carlsbad, California) was added for 8 hours at 4 degrees Celsius. MTS were then washed 3 times in 1 $\mathrm{mL}$ PBS and visualized using confocal microscopy (Zeiss Observer Z1).

\section{Results}

To study $\mathrm{Fe}_{3} \mathrm{O}_{4}$ NP penetration into MTS, we first functionalized the $10 \mathrm{~nm} \mathrm{Fe}_{3} \mathrm{O}_{4} \mathrm{NPs}$ with tumstatin. Tumstatin binds to $\alpha_{v} \beta_{3}$ integrin, which is highly expressed on the cell surface of proliferative, neovascular endothelial cells and in some cancerous cells [23]. This naturally occurring peptide has shown to significantly suppress endothelial cell proliferation in vitro by inhibiting protein synthesis [18], as a result of its action on anti-angiogenic and pro-apoptotic pathways.

\section{Preparation and characterization of tum- statin- $\mathrm{Fe}_{3} \mathrm{O}_{4} \mathrm{NP}$}

$\mathrm{Fe}_{3} \mathrm{O}_{4}$ NPs were synthesized through high temperature decomposition of $\mathrm{Fe}(\mathrm{acac})_{3}$, which allows for the precise control of particle size and quality that provides higher $T_{2} / T_{2}{ }^{*}$ relaxivity properties[24]. The as-synthesized NPs were made water-soluble through ligand exchange with a synthesized DPA-PEG-COOH polymer accomplished via our previous protocol [21]. Tumstatin was modified with Rhod before conjugation with NPs to allow for quantification and fluorescent visualization. The conjugation of tumstatin onto $\mathrm{Fe}_{3} \mathrm{O}_{4}$ NPs was achieved through EDC-NHS coupling, which allows the conjugation of $\sim 4$ tumstatin molecules on one single NP (quantified through fluorescence intensity). The prepared tumstatin- $\mathrm{Fe}_{3} \mathrm{O}_{4} \mathrm{NPs}$ were dispersed in PBS and had a hydrodynamic diameter of about $30 \mathrm{~nm}$. Figure 1 shows the TEM images of the NPs before and after tumstatin coupling, from which we observed that surface treatments did not change the NP morphology. The presence of tumstatin on $\mathrm{Fe}_{3} \mathrm{O}_{4}$ NPs was confirmed through IR (Supplementary Material: Fig. S1). Tumstatin conjugation enhanced the uptake of $\mathrm{Fe}_{3} \mathrm{O}_{4} \mathrm{NPs}$ by $\mathrm{a}_{\mathrm{v}} \beta_{3}$ integrin-expressing endothelial cells, BPAE cells. As shown Figure 2A, BPAE cells showed more than twice as much uptake of tumstatin- $\mathrm{Fe}_{3} \mathrm{O}_{4} \mathrm{NPs}$ compared with the more rapidly proliferating RG2 cells at 0.1 $\mathrm{mg} \mathrm{Fe} / \mathrm{mL}$ media concentration. The slowly prolifer- 
ating BPAE cells had comparable uptake at lower concentrations of 0.01 and $0.001 \mathrm{mg} \mathrm{Fe} / \mathrm{mL}$ media.

Both tumstatin- $\mathrm{Fe}_{3} \mathrm{O}_{4} \mathrm{NPs}$ and $\mathrm{Fe}_{3} \mathrm{O}_{4} \mathrm{NPs}$ were nontoxic to the cells studied as measured by the MTT viability assay under three concentrations $(0.1,0.01$, and $0.001 \mathrm{mg} \mathrm{Fe} / \mathrm{mL}$ ) (Figure 2B). However, proliferation of BPAE cells was inhibited by tumstatin- $\mathrm{Fe}_{3} \mathrm{O}_{4}$
NPs $(0.1 \mathrm{mg} \mathrm{Fe} / \mathrm{mL}$ ) (Figure $2 \mathrm{C}$ ) as expected, given that tumstatin is an inhibitor of endothelial cell proliferation. BPAE cell number remained relatively constant after 8 days of culture in the presence of tumstatin- $\mathrm{Fe}_{3} \mathrm{O}_{4} \mathrm{NPs}$, whereas in the presence of control $\mathrm{Fe}_{3} \mathrm{O}_{4}$ NPs cell number increased by 2.7 times.
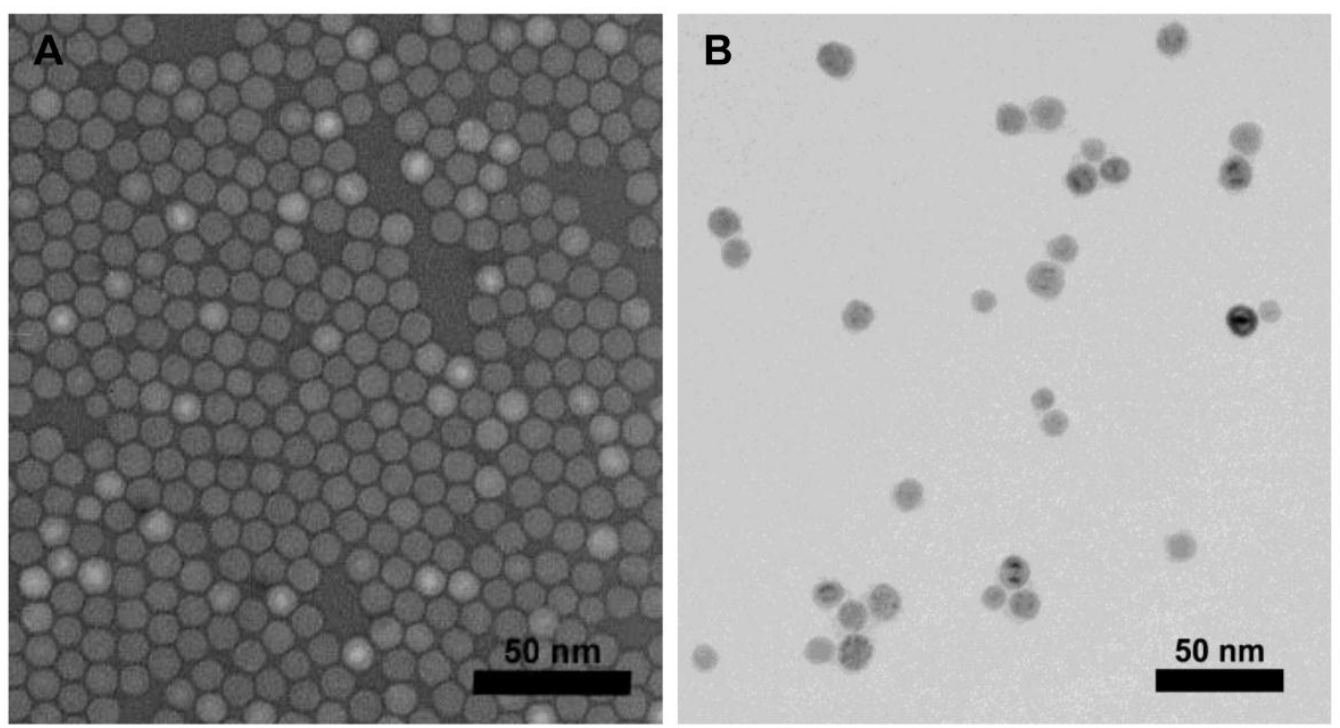

Figure I. (A) $\mathrm{Fe}_{3} \mathrm{O}_{4}$ particles as-synthesized and (B) after DPA-PEG-COOH modification and tumstatin conjugation imaged by TEM.

A

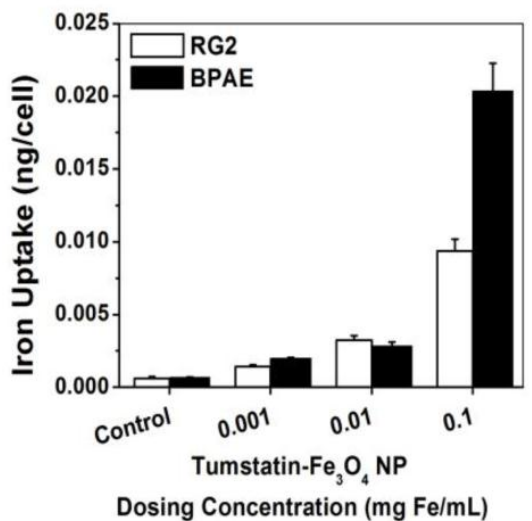

B

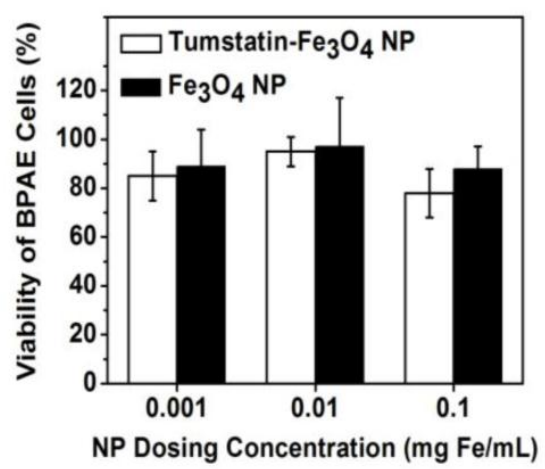

C

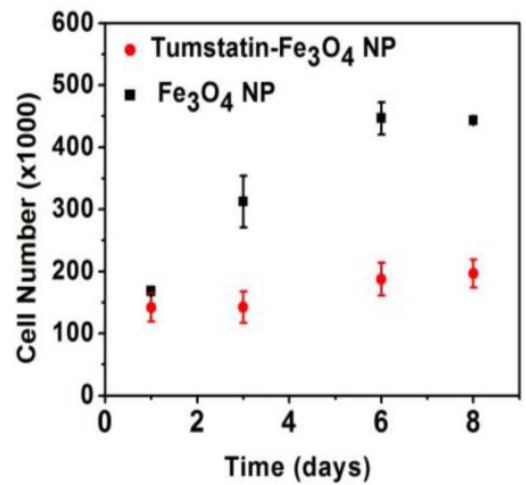

Figure 2. (A) RG2 and BPAE cells uptake more particles at a higher concentration of tumstatin- $-\mathrm{Fe}_{3} \mathrm{O}_{4} \mathrm{NP}$ as measured by elemental analysis. (B) BPAE cell viability was not significantly affected by the addition of functionalized tumstatin- $\mathrm{Fe}_{3} \mathrm{O}_{4}$ particles as compared to untargeted $\mathrm{Fe}_{3} \mathrm{O}_{4} \mathrm{NPs}$ as measured by an MTT viability assay after 24-hours of incubation. (C) Proliferation of BPAE cells decreased when incubated with tumstatin- $\mathrm{Fe}_{3} \mathrm{O}_{4} \mathrm{NPs}$ versus non-functionalized $\mathrm{Fe}_{3} \mathrm{O}_{4} \mathrm{NPs}$ $(\mathrm{Fe}=0.1 \mathrm{mg} / \mathrm{ml})$. 


\section{Fabrication and characterization of endotheli- al-coated MTS}

To construct the endothelial-coated MTS, two main conditions needed to be satisfied: the formation of MTS core, and the selection of endothelial cell line for the construction of endothelium.

Thus, we first identified the seeding conditions and growth trend of the single cell line RG2 MTS in our base microtissue design method (Supplementary Material: Fig. S2) [16]. Following the initial cell seeding process, there were two stages in the formation of MTS: cell self-assembly and cell growth. The self-assembly occurred within 24 hours right after cell seeding. Once the cell suspension was added into the agarose hydrogel, monodisperse cells settled into the non-adhesive wells, formed cell-cell contacts, and became 3D spheroids. The size of the MTS could be controlled by the seeding number of RG2 cells, thus increasing the number of cells resulted in the formation of spheroids of larger diameter (Supplementary Material: Fig. S3A). For example, at the seeding density of 822,000 cells per hydrogel, RG2 MTS assembled at a diameter of $100 \mu \mathrm{m}$. The MTS size was dispersed in a Gaussian distribution for each seeding density (Supplementary Material: Fig. S3B). In the following growth stage, the size of MTS increased as a result of RG2 cells proliferation that was revealed by the measurement of metabolic activity (Supplementary Material: Fig. S4).

After the identification of seeding density and growth pattern of the RG2 MTS (core), the next key question is to find a suitable endothelial cell line to develop the endothelium on RG2 MTS. We studied two endothelial cell lines, BPAE and human umbilical vein endothelial cells (HUVEC). Before seeding onto the agarose gels and to facilitate their identification, cells were labeled with fluorescent marker CellTracker. BPAE or HUVEC cells are shown in blue while the MTS that were formed with RG2 cells are shown in green. 24 hours after cell seeding, BPAE cells assembled an endothelium layer on the RG2 MTS, while HUVEC did not coat the MTS. HUVEC cells formed an independent spheroid adjacent to the RG2 MTS (Supplementary Material: Fig. S5). The formation of an independent HUVEC spheroid instead of the formation of an endothelium layer on the RG2 MTS is probably due to stronger HUVEC-to-HUVEC cell interaction as opposed to HUVEC-to-RG2 interactions. The importance of cell line selection was also seen when we tested MTS formed from 9L cells, another glioma cell line. BPAE cells mixed with the 9L cells indiscriminately whereas HUVEC formed small aggregates on the 9L MTS (Supplementary Material:
Fig. S5). Although greater versatility with more cell lines is desirable, cell-cell interactions dominate the formation of microtissue structures [23]. Tissue engineering and microtissue formation are continuously being studied to gain greater understanding and obtain greater control. The endothelial cell-coated glioma is a novel microtissue of interest.

After addressing the above two key components, we focused on the RG2 and BPAE model for the remainder of this study due to the desirable endothelial cell-coated glioma MTS formation. Figure 3A shows a smooth RG2 MTS as compared to the cobblestone morphology of BPAE cells when grown individually; the co-culture of BPAE and RG2 cells show a blend between the two structures of a cobblestone surface with smooth core when cultured together. BPAE cells preferentially coat the outside of the RG2 core becoming a tumor core with an endothelial cell shell after 24 hours of self-assembly (Figure 3B\&C). The use of non-adhesive, micromolded wells enabled the generation of large numbers of uniform sized spheroids. To examine their structure in more detail, BPAE-coated RG2 MTS were examined using fluorescent, confocal, and SEM after 24-hour of self-assembly. As revealed through Figure 3D, BPAE cells began to form a coating of 1-3 rounded, cobble-stone endothelial cells (resembling that of a leaky vasculature network) on top of a smooth RG2 core. As a marker for endothelium formation, tight junction markers of zona occludens 1 (ZO-1) and occludin were identified by immuno-staining and were observed to co-localize at BPAE cell-cell contacts (Supplementary Material: Fig. S6). Actin cytoskeleton staining co-localized to the cell-cell contacts and correlated with the tight junction contacts.

To quantify the extent of endothelial coating and determine the length of time the coating persisted on the RG2 MTS, we used image analysis of confocal images to measure the amount of continuous endothelial perimeter. The MTS had an endothelial cell-coating with approximately $90 \%$ and $85 \%$ coverage for days 1 and 2, respectively, after assembly (Supplementary Material: Fig. S7). Although coverage was not complete, the 1-3 layers of endothelial cells on the surface created a tortuous network in the endothelial cell coating. On day 3 , endothelial cell coverage decreased to approximately $60 \%$ due to the proliferation of the RG2 cells.

\section{Penetration study of tumstatin- $\mathrm{Fe}_{3} \mathrm{O}_{4} \mathrm{NPs}$ of endothelium-coated MTS}

The BPAE-coated RG2 MTS were incubated with tumstatin- $\mathrm{Fe}_{3} \mathrm{O}_{4}$ NPs $(0.1 \mu \mathrm{g} \mathrm{Fe} / \mathrm{mL})$ for 4 hours. $\mathrm{Fe}_{3} \mathrm{O}_{4} \mathrm{NPs}$ labeled with Rhod and not conjugated with 
tumstatin were used as a control (Supplementary Material: Fig. S8). After washing with PBS to remove the unattached NPs, MTS were examined with confocal fluorescence microscopy. As shown in Figure $4 \mathrm{~A}$, the tumstatin- $\mathrm{Fe}_{3} \mathrm{O}_{4} \mathrm{NPs}$ (red) co-localized with the BPAE cells (blue) in the top left and bottom left panel, respectively. A close-up view of the interface between BPAE and RG2 MTS (Figure 4B) confirmed that tumstatin- $\mathrm{Fe}_{3} \mathrm{O}_{4}$ NPs preferably bound onto BPAE cells even though some RG2 cells were adjacent to BPAE cells (yellow arrow in Figure 3D bottom). This specific binding indicates that tumstatin- $\mathrm{Fe}_{3} \mathrm{O}_{4}$ NPs could act as both a targeting agent to endothelium and a diagnostic to examine tumor vascularization with the help of MRI. Further studies are under way to help assess this possibility.

The BPAE-RG2 MTS labeled with tumstatin- $\mathrm{Fe}_{3} \mathrm{O}_{4} \mathrm{NPs}$ were further examined with TEM. Given that there was a BPAE coating on the RG2 core
(Figure 5A), we identified the interface between the coating and the core by the different morphologies of the BPAE cells and the RG2 cells (Figure 5B). In BPAE cells, the close-up TEM view revealed the presence of tumstatin- $\mathrm{Fe}_{3} \mathrm{O}_{4} \mathrm{NPs}$ in cell endosomes and the cytoplasm (Figure 5C\&D). However, very few tumstatin- $\mathrm{Fe}_{3} \mathrm{O}_{4}$ were observed in the core RG2 cells under TEM (Supplementary Material: Fig. S9). This observation combined with the confocal imaging results described above (Figure 4A) indicated endothelial cells limited and even blocked NPs from penetrating into the microtissue, which is similar to the tumor environment in vivo. Supported by this conclusion, Tumstatin- $\mathrm{Fe}_{3} \mathrm{O}_{4}$ should be a great candidate for targeting tumor vasculature with potential challenges in penetrating into the tumor mass. To improve the penetration of NPs through the endothelium coating and into the MTS, a higher concentration and smaller hydrodynamic size could potentially be utilized.

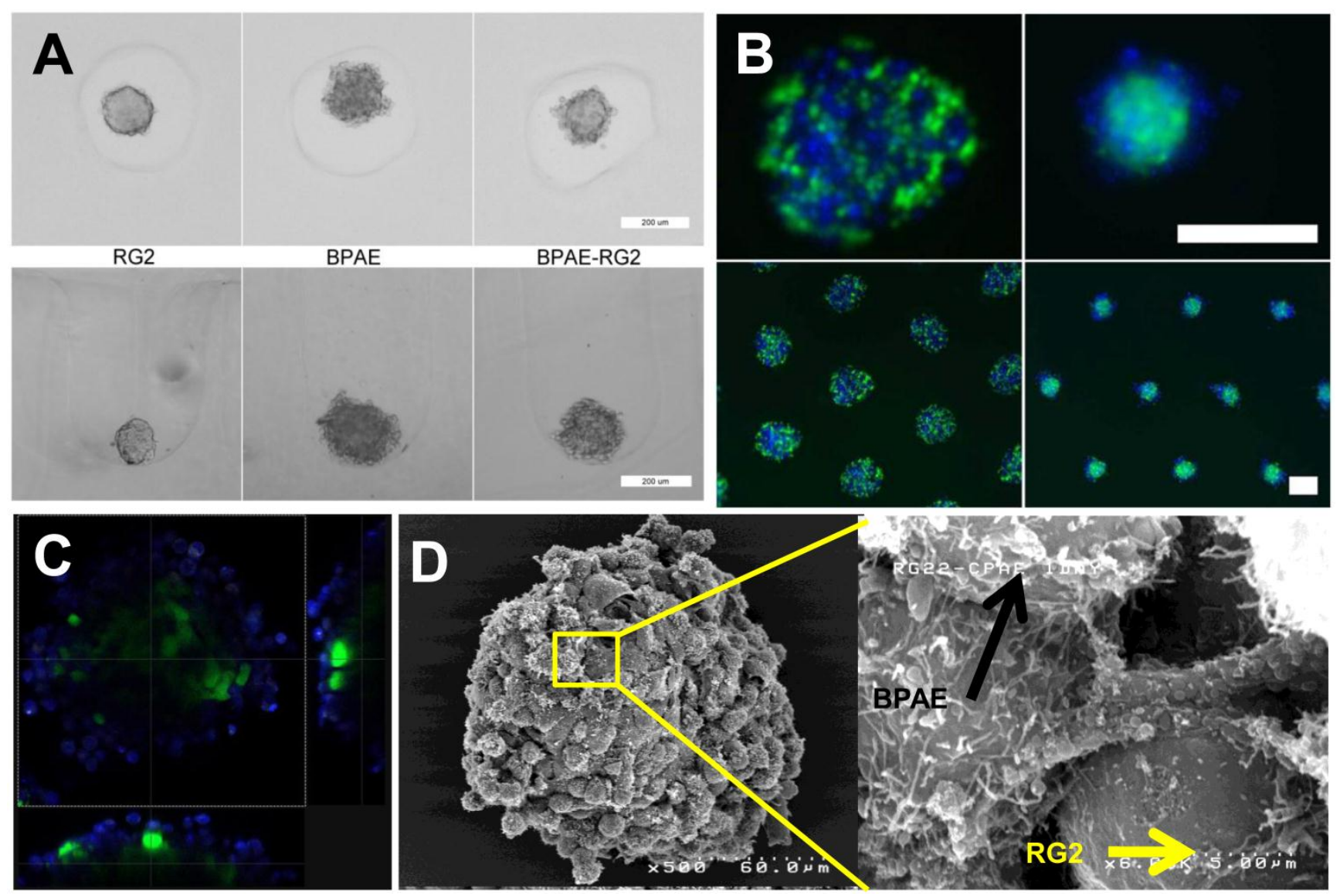

Figure 3. (A) Top-view bright field and custom side-view microscopic images of RG2 and BPAE spheroids with distinct smooth, compact and round, cobblestone morphology (scale bar $=200 \mu \mathrm{m}$ ). (B) Epifluorescence images of BPAE-RG2 MTS in agarose micro-wells show an inital mixed dispersion settling during initial assembly stage and sort to a BPAE-coated RG2 core 24 hours after cell seeding (scale bar $=200 \mu \mathrm{m}$ ). (C) Fluorescent confocal cross-section view of BPAE coverage on RG2 core after 24 hours of assembly. (D) SEM images of BPAE-RG2 MTS having BPAE cells grafted on RG2 cells. 

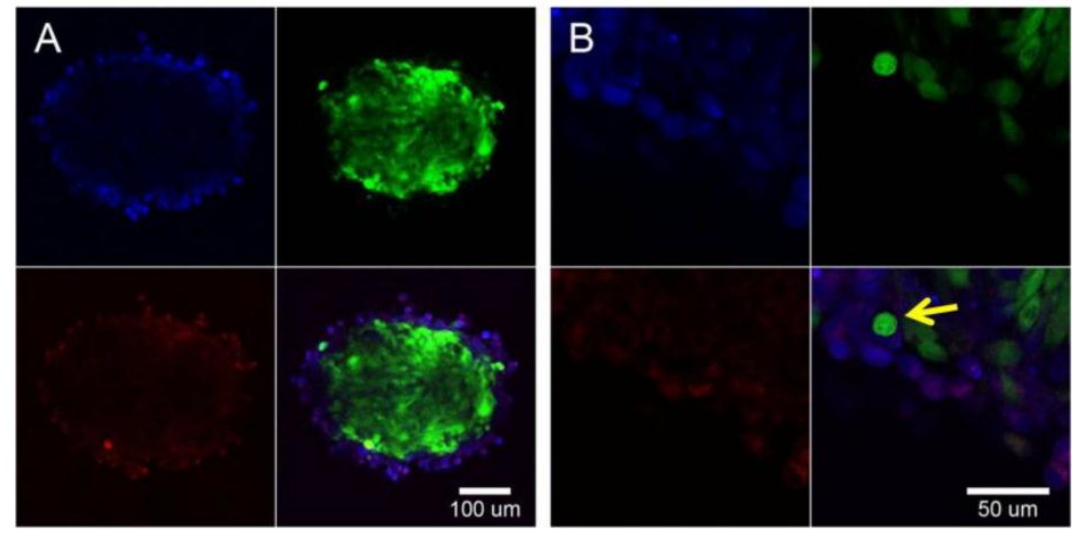

Figure 4: Tumstatin- $\mathrm{Fe}_{3} \mathrm{O}_{4} \mathrm{NPs}$ (red) selectively target BPAE endothelial cells (blue) in the heterogeneous culture with RG2 glioma cells (green). Median section of BPAE-RG2 spheroids were imaged with confocal microscopy (A). Greater magnification shows cell specificity (B) and an overlay of confocal images shows co-localization throughout the spheroid. Yellow arrow points to one RG2 cell surrounded by the BPAE cells, where BPAE cells showed fluoresce from tumstatin- $\mathrm{Fe}_{3} \mathrm{O}_{4} \mathrm{NPs}$ but RG2 cell didn't.

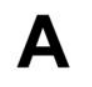

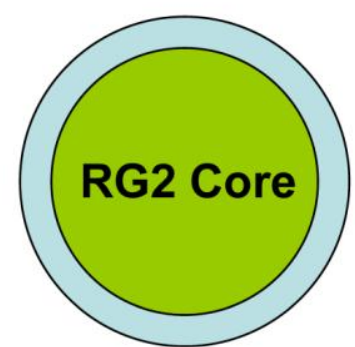

BPAE Shell
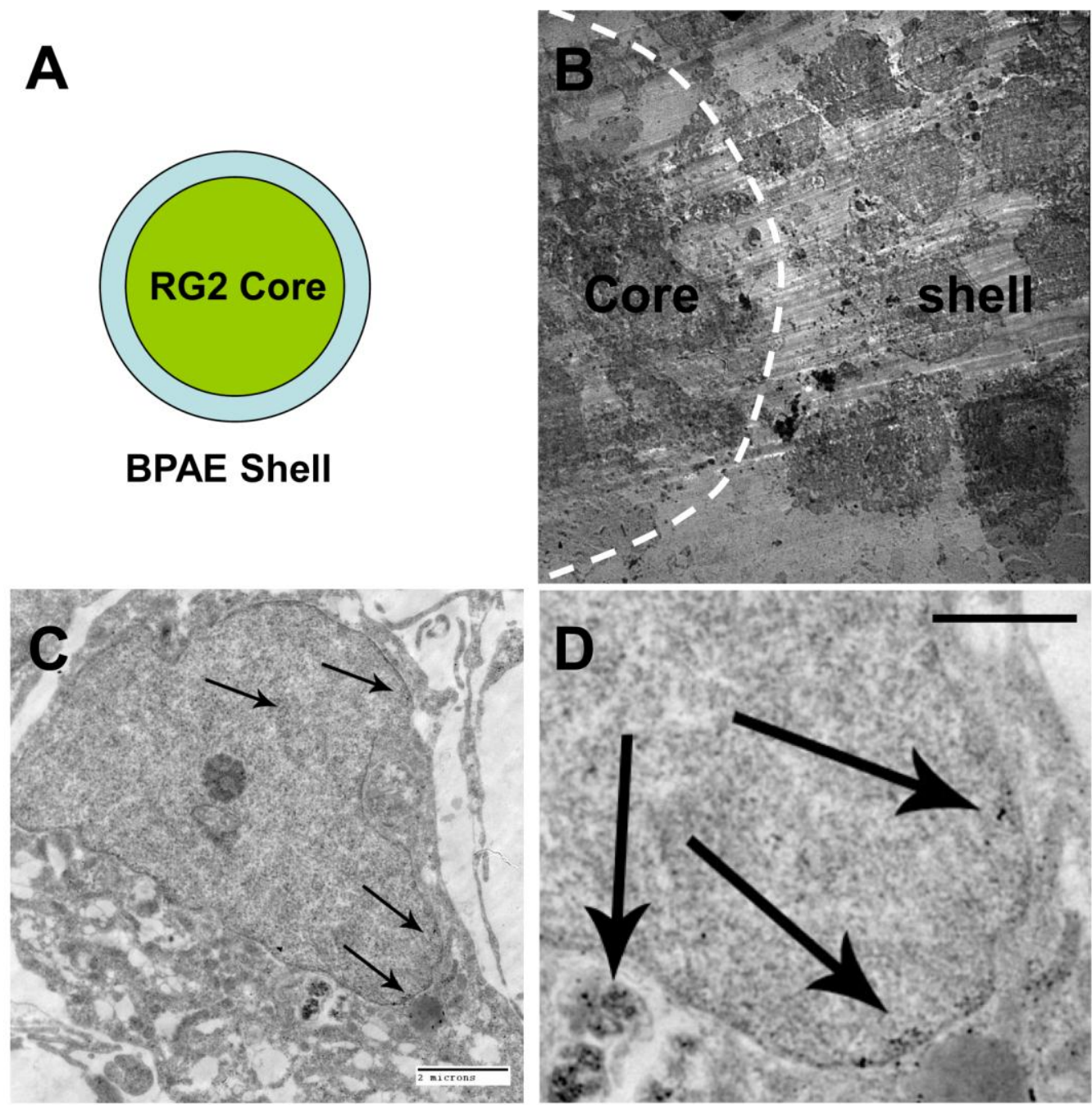

Figure 5. TEM examination of MTS labeled with Tumstatin- $\mathrm{Fe}_{3} \mathrm{O}_{4} \mathrm{NPs}$. (A) Illustration of the MTS structure; (B) image of the interface between RG2 core and BPAE shell; (C, 2 micron scale bar, and D, I micron scale bar) close examination of Tumstatin- $-\mathrm{Fe}_{3} \mathrm{O}_{4} \mathrm{NPs}$ in the endosome and cytoplasm of one BPAE cell (NPs denoted by arrows). 


\section{Discussion}

Herein, we provided a more thorough in vitro model examining the penetration ability of NPs into a tumor mass, with the aim to provide greater resolution of early detection and drug delivery. In conceiving and testing this model, two criteria guided us: the in vitro model should be similar to the in vivo microenvironment, but easy to reproduce and convenient for analysis; the NP system should be widely accepted in cancer research.

Compared with the conventional models, our endothelial-coated MTS model provides a facile and inexpensive platform between in vitro two-dimensional cell culture and the more laborious, complex and expensive animal model. The characterization of NPs in this three-dimensional in vitro model can be easily performed to examine the next incremental step before going into a more elaborate study. This model can determine the ability to penetrate a leaky tumor vasculature by diffusive properties in biological media before the complex interactions of inflammation and immune responses. Further development of this 3D microtissue model and addition of properties that more mimic the in vivo environment could help examine NPs and other theranostic agents in greater detail before applying them to an animal model. However, due to the simplicity, compared with the in vivo model, systemic interactions could not be examined such as pharmacokinetics/pharmacodynamics of NPs and also the influence of inflammatory phagocytes (tumor associated macrophages). Thus after utilizing our model to screen NPs, the more promising agents should be tested in vivo for the collection of such information.

$\mathrm{Fe}_{3} \mathrm{O}_{4}$ NPs are one of the most widely used NPs that are clinically safe and able to act as both imaging contrast agents and therapeutics. Tumstatin has been studied for its anti-angiogenic effects and mechanistic pathways have been elucidated. The in vitro test with cell culture confirmed that the conjugation of tumstatin onto $\mathrm{Fe}_{3} \mathrm{O}_{4} \mathrm{NP}$ had similar therapeutic effect, but more importantly for our application, the ability to target endothelial cells. The ability for NP penetration into a tumor with leaky vasculature is tested and examined using the 3D in vitro model. Tumstatin- $\mathrm{Fe}_{3} \mathrm{O}_{4}$ NP demonstrates this application by providing selective targeting to the leaky tumor vasculature with few NPs present in the tumor core. The increased amounts of tumstatin- $\mathrm{Fe}_{3} \mathrm{O}_{4} \mathrm{NPs}$ associated with the leaky vasculature is due to the affinity of tumstatin to the newly forming endothelial cell $\alpha_{v} \beta_{3}$ integrins. In addition to this targeting, the 1-3 endothelial cell layer coverage has begun to form an en- dothelial layer creating a torturous path similar to that found in porous media. This structure effectively increases endothelium surface area and decreases diffusion of nanoparticles as described by basic material properties of porous media and independently conducted diffusion studies (not shown). With $90 \%$ coverage at day 1 , the $10 \%$ of non-coverage is from patent and non-patent network of tortuous channels. The newly forming endothelium has the formation of tight junctions at endothelial cell contacts, but still has pores and channels on the endothelial cell layer, similar to that seen in neovascularization. Tumstatin-Fe3O4 NPs' affinity to vasculature could provide both diagnostic contrast of tumor vasculature in vivo and offer anti-angiogenic properties as shown in conventional cell culture. Greater methods are being developed to study anti-angiogenic effects in the three-dimensional model.

In summary, we have developed and characterized a 3D in vitro platform to study the penetration of NPs into tumors. By utilizing micromolded nonadhesive hydrogels, we have developed a platform composed of a multi-cellular tumor spheroid coated with an endothelium layer. The tumor spheroid mimics the solid tumor while the endothelium coating represents the endothelium of the blood vessel, a significant barrier to the delivery of therapeutics. The platform was tested with a model NP, tumstatin- $\mathrm{Fe}_{3} \mathrm{O}_{4}$ NPs that preferentially binds to endothelial cells and inhibits their proliferation. The results showed that tumstatin- $\mathrm{Fe}_{3} \mathrm{O}_{4} \mathrm{NPs}$ could specifically target to the endothelial cells in a complex 3D microtissue environment. This model has significant potential to help in the testing and optimization of the design of therapeutic/diagnostic nanocarriers and determine their therapeutic capabilities.

\section{Supplementary Material}

Fig. SI-S9. http://www.thno.org/v02p0066s I.pdf

\section{Acknowledgements}

This work was funded, in part, by NIH/NCI 1R21CA12859 (S.S), the Brown Department of Diagnostic Imaging Fund (S.S), the NIRT Program under award DMI-0506661 (J.R.M.). Partial support provided from Rhode Island Biotechnology/Biomanufacturing Training Initiative Grant.

\section{Conflict of Interest}

J.R.M. has an equity interest in MicroTissues, Inc. This relationship has been reviewed and managed by Brown University in accordance with its conflict of interest policies. 


\section{References}

1. Na HB, Song IC, Hyeon T. Inorganic Nanoparticles for MRI Contrast Agents. Advanced Materials. 2009; 21: 2133-48.

2. $\mathrm{Xu} \mathrm{CJ,} \mathrm{Sun} \mathrm{S.} \mathrm{Superparamagnetic} \mathrm{nanoparticles} \mathrm{as} \mathrm{targeted}$ probes for diagnostic and therapeutic applications. Dalton Transactions. 2009;: 5583-91.

3. Greish K. Enhanced permeability and retention (EPR) effect for anticancer nanomedicine drug targeting. Methods Mol Biol. 2010; 624: 25-37.

4. Dreher MR, Liu W, Michelich CR, Dewhirst MW, Yuan F, Chilkoti A. Tumor Vascular Permeability, Accumulation, and Penetration of Macromolecular Drug Carriers. Journal of the National Cancer Institute. 2006; 98: 335-44.

5. Minchinton AI, Tannock IF. Drug penetration in solid tumours. Nat Rev Cancer. 2006; 6: 583-92.

6. Thomlinson RH, Gray LH. The histological structure of some human lung cancers and the possible implications for radiotherapy. British Journal of Cancer. 1955; 9: 539.

7. Wong C, Stylianopoulos T, Cui J, Martin J, Chauhan VP, Jiang $\mathrm{W}$, et al. Multistage nanoparticle delivery system for deep penetration into tumor tissue. Proc Natl Acad Sci U S A. 2011; 108: 2426-31.

8. Teesalu T, Sugahara KN, Kotamraju VR, Ruoslahti E. C-end rule peptides mediate neuropilin-1-dependent cell, vascular, and tissue penetration. Proc Natl Acad Sci U S A. 2009; 106: 16157-62.

9. Tracqui P. Biophysical models of tumour growth. Reports on Progress in Physics. 2009; 72: 056701.

10. Friedrich J, Seidel C, Ebner R, Kunz-Schughart LA. Spheroid-based drug screen: considerations and practical approach. Nat Protoc. 2009; 4: 309-24.

11. Mueller-Klieser W. Multicellular spheroids. A review on cellular aggregates in cancer research. J Cancer Res Clin Oncol. 1987; 113: 101-22.

12. Waite CL, Roth CM. PAMAM-RGD Conjugates Enhance siRNA Delivery Through a Multicellular Spheroid Model of Malignant Glioma. Bioconjugate Chem. 2009; 20: 1908-16.

13. Goodman TT, Chen J, Matveev K, Pun SH. Spatio-temporal modeling of nanoparticle delivery to multicellular tumor spheroids. Biotechnol Bioeng. 2008; 101: 388-99.

14. Goodman TT, Olive PL, Pun SH. Increased nanoparticle penetration in collagenase-treated multicellular spheroids. Int J Nanomedicine. 2007; 2: 265-74.

15. Ho VHB, Slater NKH, Chen RJ. pH-responsive endosomolytic pseudo-peptides for drug delivery to multicellular spheroids tumour models. Biomaterials. 2011; 32: 2953-8.

16. Napolitano AP, Dean DM, Man AJ, Youssef J, Ho DN, Rago AP, et al. Scaffold-free three-dimensional cell culture utilizing micromolded nonadhesive hydrogels. Biotechniques. 2007; 43: 494-500.

17. Wang YX, Hussain SM, Krestin GP. Superparamagnetic iron oxide contrast agents: physicochemical characteristics and applications in MR imaging. Eur Radiol. 2001; 11: 2319-31.

18. Maeshima Y, Sudhakar A, Lively JC, Ueki K, Kharbanda S, Kahn CR, et al. Tumstatin, an endothelial cell-specific inhibitor of protein synthesis. Science. 2002; 295: 140-3.

19. Niu G, Chen X. Why integrin as a primary target for imaging and therapy. Theranostics. 2011; 1: 30-47.

20. Chen $K$, Chen X. Integrin targeted delivery of chemotherapeutics. Theranostics. 2011; 1: 189-200.

21. Xu C, Xie J, Kohler N, Walsh EG, Chin YE, Sun S. Monodisperse magnetite nanoparticles coupled with nuclear localization signal peptide for cell-nucleus targeting. Chem Asian J. 2008; 3: 548-52.
22. Youssef J, Nurse AK, Freund LB, Morgan JR. Quantification of the forces driving self-assembly of three-dimensional microtissues. Proc Natl Acad Sci U S A. 2011; 108: 6993-8.

23. Bello L, Francolini M, Marthyn P, Zhang J, Carroll RS, Nikas DC, et al. Alpha(v)beta3 and alpha(v)beta5 integrin expression in glioma periphery. Neurosurgery. 2001; 49: 380-9.

24. Hao R, Xing R, Xu Z, Hou Y, Gao S, Sun S. Synthesis, functionalization, and biomedical applications of multifunctional magnetic nanoparticles. Adv Mater. 2010; 22: 2729-42. 